

\title{
MATRIZ DE REFERENCIA PARA LA OPTIMIZACIÓN DEL CICLO DE VIDA DE LOS MATERIALES CONSTRUCTIVOS DE LA VIVIENDA SOCIAL EN ZONAS ÁRIDO-SÍSMICAS
}

\author{
A REFERENCE MATRIX FOR THE OPTIMIZATION OF THE LIFE CYCLE \\ OF SOCIAL HOUSING CONSTRUCTION MATERIALS IN ARID SEISMIC \\ ZONES.
}

ANALIA ALEJANDRA ALVAREZ Doctora Arquitecta

Instituto de Estudios en Arquitectura Ambiental (INEAA):

Facultad de Arquitectura, Urbanismo y Diseño (FAUD); Universidad Nacional de San Juan (UNSJ), San Juan, Argentina https://orcid.org/0000-0003-0069-8173 ana_alv023@hotmail.com
VERONICA RIPOLL MEYER

Arquitecta

Instituto de Estudios en Arquitectura Ambiental (INEAA); Facultad de Arquitectura, Urbanismo y Diseño (FAUD); Universidad Nacional de San Juan (UNSJ), San Juan, Argentina veronicaripoll@yahoo.com

\section{RESUMEN}

El objetivo de esta investigación es contribuir con la sustentabilidad ambiental en el árido a través del desarrollo de un sistema de apoyo a la toma de decisiones en las distintas fases que intervienen en el ciclo de vida (ACV) de los edificios. Para ello, se analizan los materiales característicos de los sistemas constructivos racionalizados propios de zonas árido-sísmicas en relación con las emisiones de dióxido de carbono asociadas a los mismos durante su producción, uso y disposición final. Los resultados alcanzados permiten determinar el nivel de sustentabilidad de los materiales analizados de acuerdo con su importancia relativa en la cadena de valor de la construcción de un país emergente como Argentina. Con base en ello, puede realizarse la estimación temprana del comportamiento energético de la vivienda social, así como la optimización de dichos materiales conforme a una mirada integral de la problemática habitacional en términos de ciclo de vida. Por tanto, la herramienta desarrollada permite empoderar a los usuarios de dichas viviendas, para que puedan ejercer su derecho a una vivienda digna y a un ambiente sano, equilibrado y apto para el desarrollo humano, tal como lo establecen los artículos 14bis y 41 de la Constitución Nacional Argentina.

Palabras clave

ciclo de vida, materiales constructivos, vivienda social, zonas árido-sísmicas.

\section{ABSTRACT}

The objective of this research is to contribute to environmental sustainability in arid zones through the development of a support system for decision making in the different stages in the life cycle (LC) of buildings. To this end, the materials characteristic of the streamlined construction systems of arid seismic zones were analyzed in relation to the associated carbon dioxide emissions during their production, use and final disposal. The results made it possible to determine the level of sustainability of the materials analyzed according to their relative importance in the value chain of construction in an emerging country such as Argentina. Based on this, an early estimation of the energy behavior of social housing can be made, as well as the optimization of said materials according to a comprehensive view of the housing problem in terms of life cycle. Therefore, the tool developed empowers the users of these dwellings, so that they can exercise their right to decent housing and a healthy, balanced and suitable environment for human development, as established in Articles 14bis and 41 of the Argentinian National Constitution.

Keywords 


\section{INTRODUCCIÓN}

Las transformaciones vinculadas al cambio en el uso del suelo han dado lugar al incremento de las emisiones de dióxido de carbono y, en consecuencia, al cambio climático (Carpinetti y Esponda, 2013). Garrido Piñero (2015) destaca que la incorporación de la dimensión urbana dentro de la construcción del paradigma del desarrollo sustentable ha cobrado importancia como resultado de las amenazas ambientales asociadas a la alta densidad poblacional y sus respectivos patrones de consumo.

En este contexto, Zabalza Bribían et al. (2014) expone que el uso masivo de materiales generalizados a nivel mundial ha causado un incremento notable de los costes energéticos y ambientales de cualquier tipología edilicia. Es decir, el impacto ambiental global del edificio depende de la elección de los materiales para su construcción. Por ello, Carabaño et al. (2017) considera necesaria la caracterización de dichos materiales a nivel medioambiental. En este sentido, Corona Bellostas (2016) sugiere que contar con herramientas que permitan cuantificar el nivel de sustentabilidad de los productos y servicios es fundamental para que la actividad humana se desarrolle beneficiosamente, tanto para la sociedad como para la preservación del ambiente.

El análisis del ciclo de vida (ACV) aplicado a productos de construcción y edificios, proporciona información imprescindible para la evaluación de la sustentabilidad ambiental de los mismos. Si bien la fase de uso tiene un gran peso en términos de impacto energético y de emisiones de gases de efecto invernadero, existen grandes oportunidades para la reducción de impactos en las fases anteriores del ciclo de vida (Villar-Burke, 2014). Basbagill et al. (2013) y Röck et al. (2018) sostienen, en tal sentido, que la realización del ACV en etapas iniciales de diseño permite alcanzar un panorama completo de los impactos ambientales vinculados a la construcción.

Con base en lo antedicho, resulta fundamental garantizar la renovación económica y medioambiental de las viviendas sociales, para alcanzar los compromisos internacionales relativos al cambio climático (López-Mesa et al., 2013). En esa dirección, implementar el Cradle to Cradle como paradigma de desarrollo socioeconómico de diseño, implica considerar todos los materiales involucrados en el proceso como nutrientes (Ros García y Sanglier, 2017). Para ello, es preciso incorporar sistemas de "logística inversa", cuyo objetivo sea la recuperación de los materiales de una manera efectiva y eficiente. Por consiguiente, es ineludible que gobiernos y agencias ambientales elaboren directrices o códigos de construcción y políticas ambientales idóneas (Carabaño, Bedoya y Ruiz, 2014).

Desde este enfoque, se espera que los resultados obtenidos hagan posible optimizar, a partir de la estimación temprana del impacto en el cambio climático de la solución constructiva adoptada, los procesos que intervienen en los modelos de ciclo de vida de los distintos materiales que conforman los sistemas constructivos racionalizados de la oferta edilicia característica de la vivienda social, en zonas árido-sísmicas.

\section{MATERIALES Y MÉTODOS}

Se define como material sustentable aquel cuyo proceso de extracción, manufactura, operación y disposición final tiene un impacto ambiental bajo o inexistente (sustentabilidad ambiental). Asimismo, su elaboración y distribución es económicamente viable (sustentabilidad económica), en tanto su vida útil no compromete la calidad de vida de los seres vivos que están de alguna manera relacionados con él (sustentabilidad social) (Delgado Castillo y Velázquez Flores, 2012). La Tabla 1 muestra el nivel de sustentabilidad de los distintos componentes arquitectónicos seleccionados para el análisis. Se pone en valor su uso en la materialización de la vivienda de interés social, su importancia relativa en la cadena de valor de los materiales de la construcción en un país en vías de desarrollo como Argentina y los impactos generados durante su producción. Como resultado, se obtiene que los materiales típicos de un sistema constructivo tradicional racionalizado en una zona árida como la ciudad de San Juan presenten un nivel medio de sustentabilidad.

Con base en las normas ISO 14040/2006', ISO 14044/2006 ${ }^{2}$ e IRAM 21931-1/12, en los siguientes apartados A, B, C y D se expone la forma en que la metodología del ACV es abordada en esta investigación.

A) OBJetivo Y ALCANCE. Muñoz y Quiroz (2014) señalan que las características del sector de la construcción dificultan la elaboración de un análisis completo de los productos que intervienen en su materialización. Marrero et al. (2013), por su parte, exponen que, tanto el impacto energético como las emisiones de $\mathrm{CO}_{2}$ asociadas a algunas soluciones constructivas, pueden valorarse a partir de la cantidad de energía utilizada para su generación. Para Ali et al. (2015), el calentamiento global es uno de los impactos

[1] https://web.stanford.edu/class/cee214/Readings/ISOLCA.pdf

[2] http://wap.sciencenet.cn/home.php?mod=attachment\&id=4637

[3] De acuerdo con Chacón y D'amelio (2015), se entiende por huella de carbono a la cantidad de CO2 que se genera de tal compuesto en un determinado proceso o actividad. 


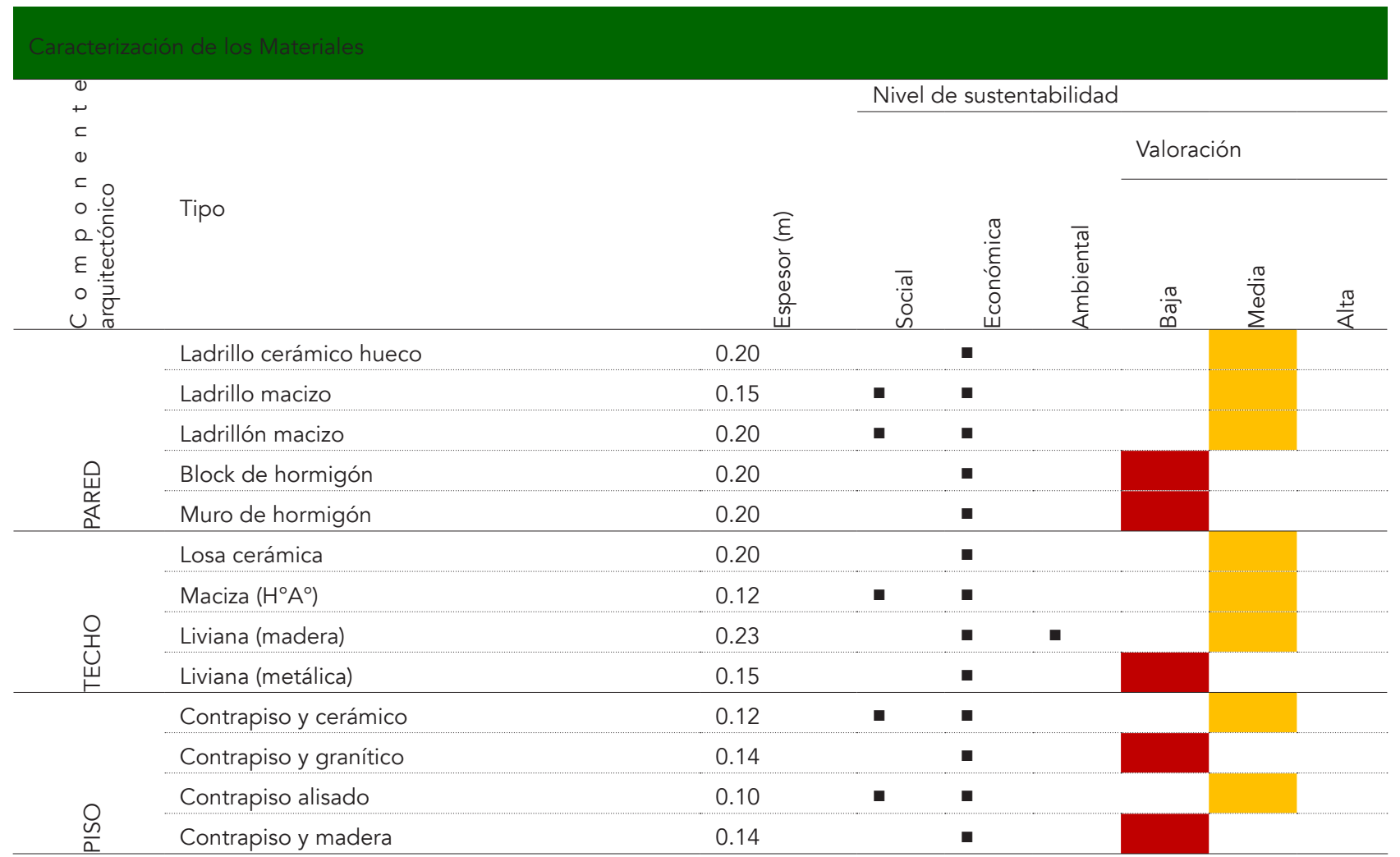

Referencias: a Sustentabilidad asociada

con mayor influencia negativa sobre el ambiente. En este contexto, se propone la realización de un análisis del ciclo de vida simplificado. De esta forma, se circunscribe el estudio a la cuantificación de la huella de carbono ${ }^{4}$ de los materiales involucrados en la concreción de viviendas sociales en zonas árido-sísmicos, a partir de la adopción de los siguientes criterios de alcance:

- Límite espacial: viviendas sociales del Área Metropolitana de San Juan (AMSJ) desarrolladas por el Instituto Provincial de la Vivienda repartición San Juan entre 2010 y 2015.

- Límite temporal: de acuerdo con la IRAM 21931-1/12, el ACV propuesto corresponde a una mirada denominada: "de la fábrica a la tumba".

- Unidad funcional: se adopta el metro cuadrado $\left(\mathrm{m}^{2}\right)$, se considera una vida útil de 50 años y se orienta el estudio a aquellos actores (decisores y ocupantes) que intervienen en el proceso de construcción del hábitat social.

- Limitaciones: quedan fuera del análisis la etapa de extracción y procesamiento de la materia prima. Para el cálculo de la energía incorporada por elemento y de acuerdo con el material que se adopte, se considera que:

1. El transporte (puesto de venta - obra) es realizado por un camión eje simple $4 \times 2$, cuya carga útil es de 7 toneladas y consumo estimado, de $6 \mathrm{~km} / \mathrm{l}$ de gasoil.

2. La distancia promedio desde el punto de venta a la obra es de 4,34 km. La misma se estima considerando las características de la trama urbana AMSJ.

3. Los consumos de energía durante la etapa previa a la entrega se obtienen de fuentes primarias de información.

4. Los consumos de energía en la etapa posterior a la entrega, para climatización, se calculan conforme a las normas IRAM 11604 (calefacción) e IRAM 116591 y 2 (refrigeración). Con base en las características urbanas del AMSJ, para la estimación de las emisiones resultantes de transporte en fase de uso, se contempla que, mínimamente, se realizan cuatro viajes al centro de la ciudad por día.

Usuarios: determinación, participación y propósito se detallan en la Tabla 2. 


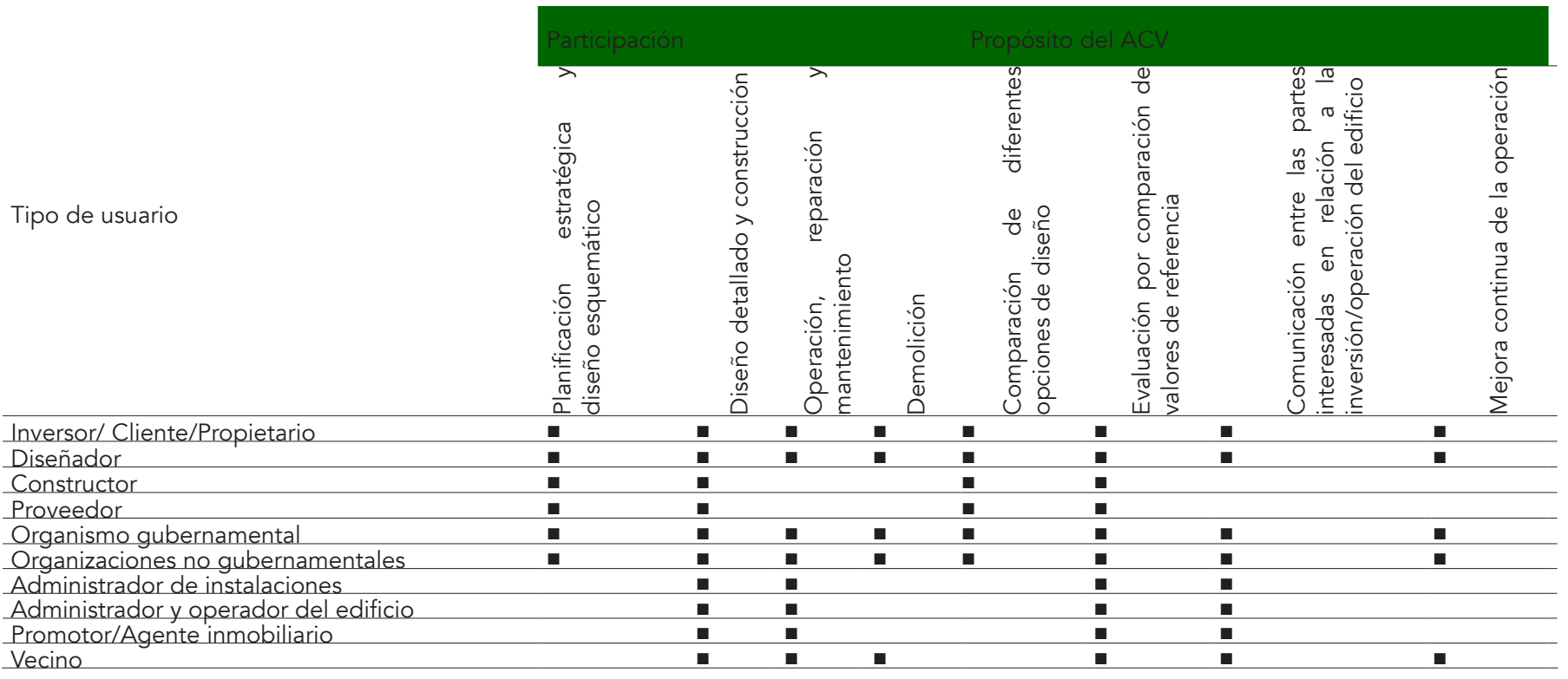

Referencias: variable asociada al actor

Tabla 2. Usuarios del ACV - Participación y Propósito Fuente: Elaboración de las autoras.

B) ANÁLISIS DEL INVENTARIO. De acuerdo con el apartado A, esta investigación se corresponde con la cuantificación del $\mathrm{CO}_{2}$ generado durante la fabricación de los distintos materiales de uso generalizado en la construcción de la vivienda social en zonas árido-sísmico, así como también las emisiones asociadas a la etapa de uso y fin de vida de la misma.

Desde esa perspectiva, se coteja lo que Quispe Gamboa (2016) denomina la "energía incorporada" ("El") (etapa previa) y la "energía operativa" ("EO") (etapa de uso). La energía incorporada corresponde con la energía consumida para el transporte y fabricación de los componentes arquitectónicos por cada metro cuadrado de construcción de los mismos. La energía operativa se extrae de la consideración conjunta de energía necesaria para calefacción y refrigeración y de las emisiones asociadas al transporte durante la vida útil de la vivienda. La etapa de fin de vida se mide a partir de las emisiones asociadas al transporte de los residuos de la construcción y demolición, desde la obra hasta el vertedero.

Con base en lo anterior, la cuantificación del $\mathrm{CO}_{2}$ se obtiene de:

Emisiones totales de vivienda $=\mathrm{El}_{\mathrm{T}}+\mathrm{EO}+\mathrm{T}_{\mathrm{DF}}\left[\mathrm{Kg} \mathrm{CO} \mathrm{KO}_{2}\right.$ eq $\left./ \mathrm{kWh}\right]$

$\mathrm{El}_{\mathrm{T}}=\left(\mathrm{EI}_{\mathrm{TPM}}{ }^{\star} \mathrm{FE}\right)+\left(\mathrm{EI}_{\mathrm{TTM}}{ }^{\star} \mathrm{FE}\right)[\mathrm{kg} \mathrm{CO} \mathrm{eq} / \mathrm{kWh}]$
$\mathrm{EO}=\left(\mathrm{E}_{r}^{*} \mathrm{FE}\right)+\left(\mathrm{E}_{c}^{*} \mathrm{FC}{ }^{\star} \mathrm{FE}\right)+\mathrm{T}[\mathrm{kg} \mathrm{CO} 2 \mathrm{eq} / \mathrm{kWh}]$

$\mathrm{T}_{\mathrm{DF}}=\left(\mathrm{C}_{\mathrm{cc}}{ }^{*} \mathrm{FC} \mathrm{C}^{\star} \mathrm{FE}\right)^{\star} \mathrm{C}_{\mathrm{VFD}}[\mathrm{kg} \mathrm{CO} 2 \mathrm{eq} / \mathrm{kWh}] \mathrm{El}_{\mathrm{TPM}}=\sum \mathrm{El}_{i}{ }^{*} \mathrm{~m}_{\mathrm{i}}[\mathrm{kWh}]$

$\mathrm{El}_{\text {Tтм }}=\mathrm{Com}_{\text {nec }} * \mathrm{FC}[\mathrm{kWh}]$

$\mathrm{T}=\left[\left(\mathrm{C}_{\mathrm{ca}}{ }^{*} \mathrm{C}_{\mathrm{vd}}{ }^{\mathrm{nec}}{ }^{*} \mathrm{~V}_{\mathrm{u}}\right]^{\star} \mathrm{FC} * \mathrm{FE}[\mathrm{kg} \mathrm{CO} 2 \mathrm{eq} / \mathrm{kWh}]\right.$

$\mathrm{Com}_{\text {nec }}=\mathrm{C}_{\mathrm{cc}}{ }^{*} \mathrm{C}_{\mathrm{v}}[\mathrm{l}]$

$\mathrm{C}_{\mathrm{V}}=\mathrm{Cap}_{\mathrm{CT}} / \mathrm{Sup}_{\mathrm{MT}}$

$\mathrm{C}_{\mathrm{VFV}}=$ volumen vivienda/volumen contenedor

$\mathrm{Cap}_{\mathrm{CT}}=\mathrm{C}_{\mathrm{Cac}} /\left(\right.$ masa* $^{*}$ Sup $\left._{\mathrm{MT}}\right)\left[\mathrm{m}^{2}\right]$

$C_{c a}=D c / A_{a}[l]$

$C_{c c}=D / A_{c}[l]$

$C_{\mathrm{vd}}^{\mathrm{cc}}: \mathrm{D}_{\mathrm{L}}^{*} \mathrm{~V}_{\mathrm{dp}}$

Donde:

A : Autonomía automóvil familiar $\left(0.734 \mathrm{~km} / /^{5}\right)$.

A: Autonomía camión (6 km/l).

$\mathrm{C}_{\mathrm{ca}}$ : Consumo combustible automóvil familiar.

$\mathrm{C}_{\mathrm{cc}}$ : Consumo combustible camión.

$\mathrm{C}_{\text {cac }}$ : Capacidad de carga admisible del camión (7000 kg).

Cap $_{C T}$ : Capacidad de carga del camión en $\mathrm{m}^{2}$ a transportar. Com $_{\text {nec }}$ : Combustible necesario para el trasporte de materiales al punto de venta/obra.

$C_{v}$ : Cantidad de viajes necesarios para transportar el material a la obra.

$\mathrm{C}_{\mathrm{vd}}$ : Cantidad de viajes diarios.

$\mathrm{C}_{\mathrm{VFV}}$ : Cantidad de viajes necesarios para transportar el material al vertedero'.

D: Distancia para transportar el material a la obra/vertedero.

[5] Corresponde al promedio de la autonomía de los vehículos familiares más vendidos. Fuente: http://www.lavoz. com.ar/autos/los-que-gastan-menos.

[6] Volumen aproximado contenedor $5 \mathrm{~m} 3$. 


\begin{tabular}{llllll} 
Combustible & $\begin{array}{l}\text { Unidades de medida } \\
\text { física }\end{array}$ & Factor de conversión & \multicolumn{2}{l}{$\begin{array}{l}\text { Factor de emisión } \\
\text { CO2 eq/kWh) }\end{array}$} \\
\hline Gas natural & $\mathrm{Nm} 3$ & 10,7056 & $\mathrm{kWh} / \mathrm{Nm} 3$ & 0,2016 & $\mathrm{Kg} \mathrm{de} \mathrm{CO2} \mathrm{eq/kWh}$ \\
\hline Gasóleo & $\mathrm{Litros}$ & 10,6 & $\mathrm{kWh} / \mathrm{Kg}$ de & 0,2628 & $\mathrm{Kg}$ de CO2 eq/kWh \\
\hline Electricidad & $\mathrm{kWh}$ & - & - & 0.385 & $\mathrm{Kg}$ de CO2 eq/kWh \\
\hline
\end{tabular}

Tabla 3. Factores de conversión y emisión utilizados en el estudio. Fuente: Elaboración de las autoras con base en datos del Fondo Europeo de Desarrollo Regional 7 .

Dc: Distancia de la obra al centro de la ciudad en $\mathrm{km}$.

$D_{1}$ : Días laborales anuales (aproximadamente 246).

$E_{c}$ : Energía necesaria calefacción. Se obtiene al aplicar la norma IRAM 11604 (Q)

$E_{\text {: }}$ Energía necesaria refrigeración. Surge al aplicar la norma IRAM $11659\left(\mathrm{Q}_{\mathrm{R}}\right)$

El: Energía incorporada de los elementos que componen los distintos paquetes constructivos ${ }^{8}$.

$\mathrm{El}_{\mathrm{T}}$ : Energía incorporada total.

$\mathrm{EI}_{\mathrm{TPM}}$ : Energía incorporada total para la producción de materiales.

$\mathrm{EI}_{\text {Ттм}}$ : Energía incorporada total para el transporte de materiales.

EO: Energía operativa.

FC: Factor de conversión.

FE: Factor de emisión.

Sup $_{\mathrm{MT}}$ : Superficie del material a transportar.

$T_{D F}$ : Transporte disposición final.

$\mathrm{V}_{\mathrm{dp}}$ : Viajes diarios promedio de una familia tipo (aproximadamente 4).

$\bigvee_{u}$ : Vida útil de la vivienda (50 años).

La Tabla 3 sintetiza los factores de emisión utilizados en cada caso.

C) EVALUACIÓN DE IMPACTOS. El cálculo de los impactos respectivos se realiza conforme a la siguiente expresión:

$\mathrm{CCl}=\sum \mathrm{GWP}_{i} \times \mathrm{m}_{i}\left[\mathrm{Kg} \mathrm{CO} \mathrm{CO}_{2}\right]$

Donde:

$\mathrm{CCl}^{9}$ : Indicador de cambio climático.

GWP: Potencial de calentamiento global= 1 (horizonte temporal de 100 años) ${ }^{10}$ $m_{i}$ : Masa de la substancia $i$.

Peso Unitario ${ }^{12}$ : su valor se obtiene del CIRSOC 101Reglamento Argentino de Cargas Permanentes y Sobrecargas Mínimas de Diseño para Edificios y Otras Estructuras (2005) $)^{13}$.

D) INTERPRETACIÓN. La Tabla 4 expone los componentes arquitectónicos clasificados de acuerdo con su transmitancia térmica (K). Se entiende por "recomendable" aquellas combinaciones de materiales cuyo $\mathrm{K}$ corresponde al nivel $C$ de las normas IRAM 11603, 11604 y 11605. La opción "aceptable" es aquella que se relaciona con la práctica constructiva habitual de una ciudad de clima árido como San Juan. Al incluir la transmitancia térmica del componente se hace referencia a la etapa de uso de la vivienda, como también a la etapa previa.

\section{MATRIZ DE MULTIOBJETIVO}

\section{OPTIMIZACIÓN}

Para Quispe Loyola (2017), el proceso de toma de decisiones comienza con la identificación y definición del problema y culmina con la elección de una alternativa. Cuando el número de alternativas es infinito el problema es calificado como "decisión multiobjetivo". Por el contrario, cuando el conjunto de alternativas es finito se trata de una "decisión multicriterio discreta" (Hurtado, 2005). Dentro de estos últimos se encuentra el llamado "proceso analítico jerárquico" (PAJ).

Celemín (2014) destaca que el PAJ divide una decisión compleja en un conjunto de decisiones simples, como resultado de crear una matriz cuadrada, en la que el número

[7] https://www.camarazaragoza.com/wp-content/uploads/2012/10/calculoemisiones.xls

[8] Su valor se obtiene de Quispe Gamboa (2016).

[9] Véase: Antón Vallejo (2004).

[10] IPCC (2014).

[11] El factor de conversión utilizado es: $1 \mathrm{kN} / \mathrm{m} 3=102 \mathrm{kN} / \mathrm{m} 2$. Fuente: https://www.convert-me.com/es/convert/ pressure/knmsq.html?u=knmsq\&v=1

[11] Fuente: https://www.inti.gob.ar/cirsoc/pdf/101/reglamento/Reglamento-cirsoc101-completo.pdf

[12] El factor de conversión utilizado es: $1 \mathrm{kN} / \mathrm{m} 3=102 \mathrm{kN} / \mathrm{m} 2$. Fuente: https://www.convert-me.com/es/convert/ pressure/knmsq.html?u=knmsq\&v=1

[13] Fuente: https://www.inti.gob.ar/cirsoc/pdf/101/reglamento/Reglamento-cirsoc101-completo.pdf 


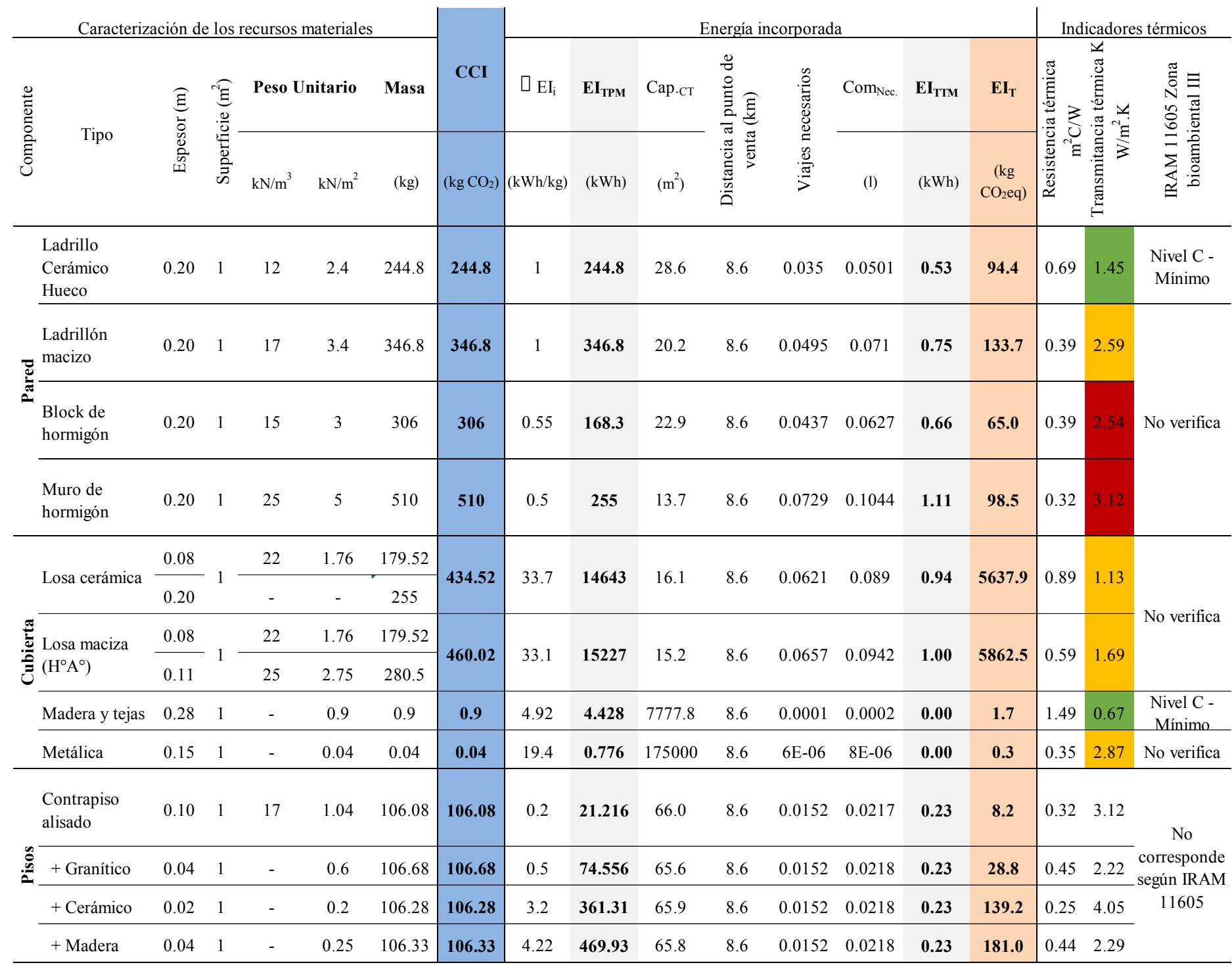

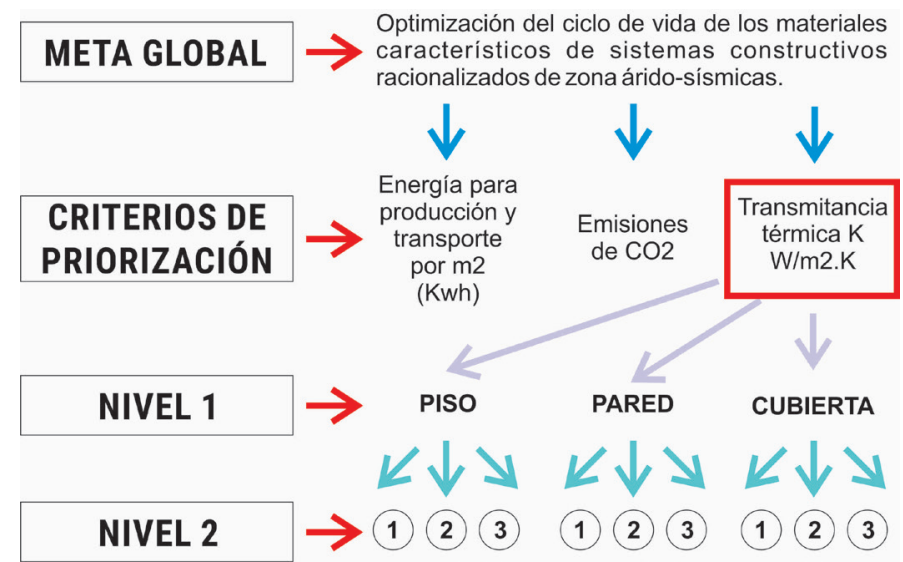




\begin{tabular}{l|l} 
Nivel 1 & $\%$ \\
\hline Piso & 33 \\
\hline Pared & 33 \\
\hline Cubierta & 33 \\
\hline
\end{tabular}

\begin{tabular}{ll|l}
\cline { 2 - 2 } Nivel 2 & $\%$ \\
Contrapiso & $\begin{array}{l}\text { y cerámico } \\
\text { y granítico } \\
\text { alisado }\end{array}$ & 8.3 \\
\hline y madera & 62.6 \\
\hline $\begin{array}{l}\text { Ladrillo cerámico hueco } \\
\text { Ladrillón macizo }\end{array}$ & 22.3 \\
Block de hormigón & 66.5 \\
Muro de hormigón & 14.2 \\
\hline Losa cerámica & 15.1 \\
Losa maciza & 4.2 \\
Madera y tejas & 12.7 \\
Metálica & 11.2 \\
\hline
\end{tabular}

Tabla 5. Resultados alcanzados en el PAJ. Fuente: Elaboración de las autoras.

MATRIZ DE OPTIMIZACIÓN MULTIOBJETIVO

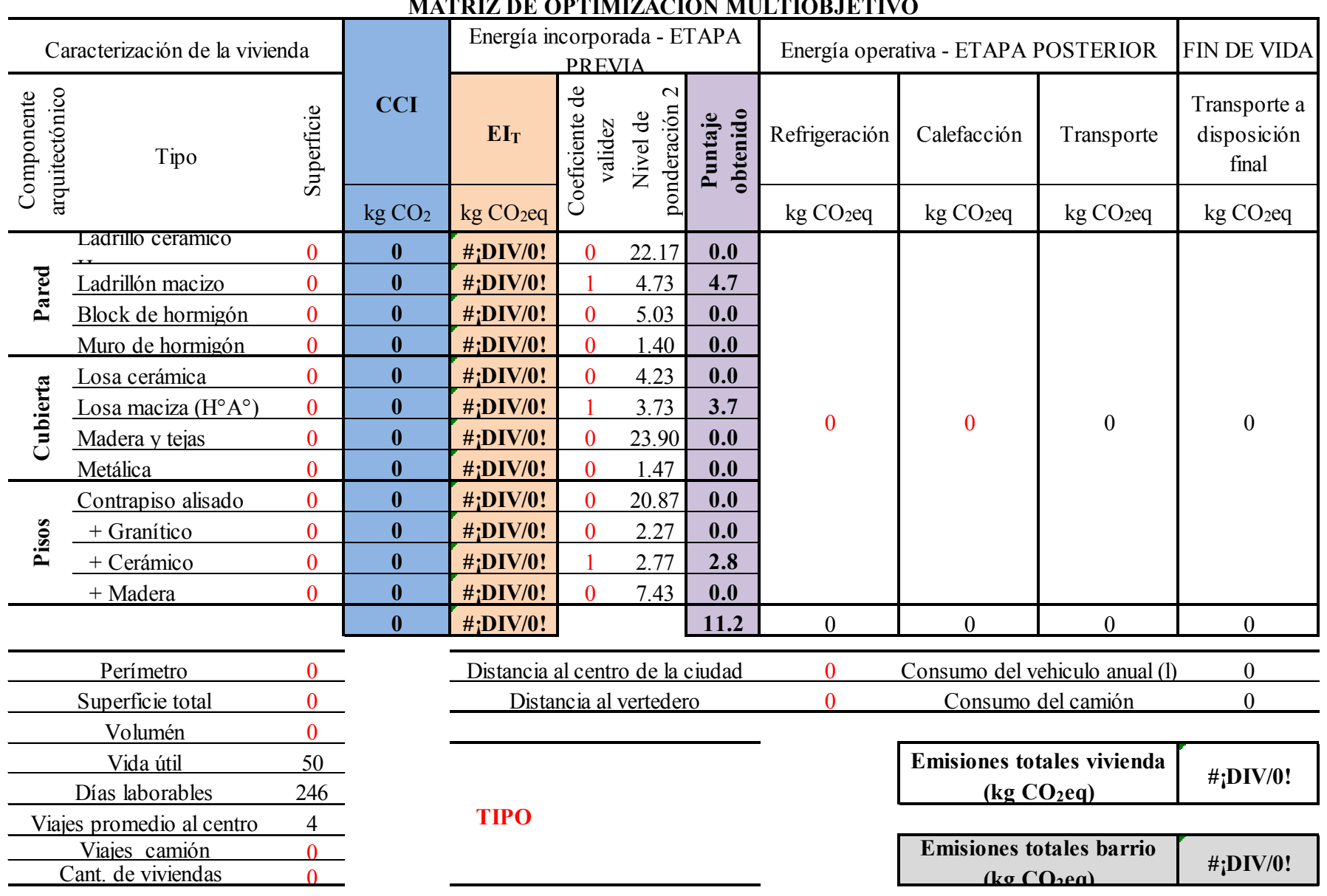

Tabla 6. Matriz de optimización multiobjetivo. Fuente: Elaboración de las autoras.

de filas y columnas viene definido por el número de criterios considerados en la toma de decisión. El uso del PAJ como herramienta orientada a la toma de decisiones resulta en decisiones justificadas que se traducen en mejores resultados en los proyectos (Vallejo-Borda, Gutiérrez y Ponz, 2014).

A partir del uso del software libre BPMSG AHP desarrollado por Klaus Goepel ${ }^{14}$, se realiza el PAJ a los componentes arquitectónicos analizados conforme al esquema que sintetiza la Figura 1. Los resultados alcanzados se muestran en la Tabla 5.

Con la ponderación de los distintos componentes constructivos característicos de los sistemas racionalizados de zonas árido-sísmicas se desarrolla la Matriz de Optimización Multiobjetivo (MOM) (Tabla 6). 
La MOM permite obtener el impacto de la vivienda social de zona árido-sísmica en relación con el cambio climático. Para ello, deberán completarse los ítems que se indican en rojo, lo cual requiere conocer las características constructivas y geométricas de la vivienda, además de su ubicación. El criterio para el llenado de la matriz es el siguiente:

- Coeficiente de validez: completar con 1 la opción que corresponda al componente constructivo considerado.

- Ponderación nivel 2: ponderaciones obtenidas en el PAJ y estandarizadas en relación con el nivel 1.

- Puntaje obtenido: sumatoria de las ponderaciones correspondientes a cada nivel.

- Tipo: este dato debe ser completado por el evaluador a partir de lo expuesto en la Tabla 7.

- Emisiones totales del barrio: surge del producto entre las emisiones de $\mathrm{CO}_{2}$ de la vivienda por la cantidad de viviendas contempladas dentro del emprendimiento urbano.

\begin{tabular}{lll}
\hline TIPO & PUNTAJE OBTENIDO & \\
\hline Recomendable & $\geq 66.93$ & \\
Aceptable o habitual & $\leq 66.92$ & $\geq 5.14$ \\
No sustentable & $\leq 5.13$ & \\
\hline
\end{tabular}

Tabla 7. Ponderación por tipo. Fuente: Elaboración de las autoras.

Dado que los puntajes están estandarizados al nivel 1, en ningún caso se obtienen valores superiores a 66.93 (Tabla 7). Ello se debe a que ninguna de las opciones posibles para los distintos componentes constructivos analizados corresponde al nivel A de la norma IRAM 11601 y 11605 (100\% del nivel). Dicha situación hace posible incorporar soluciones constructivas más sustentables. Consecuentemente, se puede afirmar que la $\mathrm{MOM}$ es dinámica y permite una valoración rápida del comportamiento ambiental de la vivienda desde la etapa de diseño.

\section{RESULTADOS}

Para la aplicación de la MOM, se toma como caso de estudio cuatro viviendas desarrolladas en el AMSJ (Figura 2). Dichas viviendas fueron ejecutadas por el IPV - San Juan entre 2010 - 2015, en los barrios Valle Grande (Rivadavia), Los Horcones (Rawson), El Prado (Chimbas) y Huarpes (Rawson). La Tabla 8 sintetiza los resultados alcanzados en la evaluación.

La Tabla 8 expone las emisiones de $\mathrm{CO}_{2}$ totales a lo largo de toda la vida útil de las viviendas evaluadas, así como

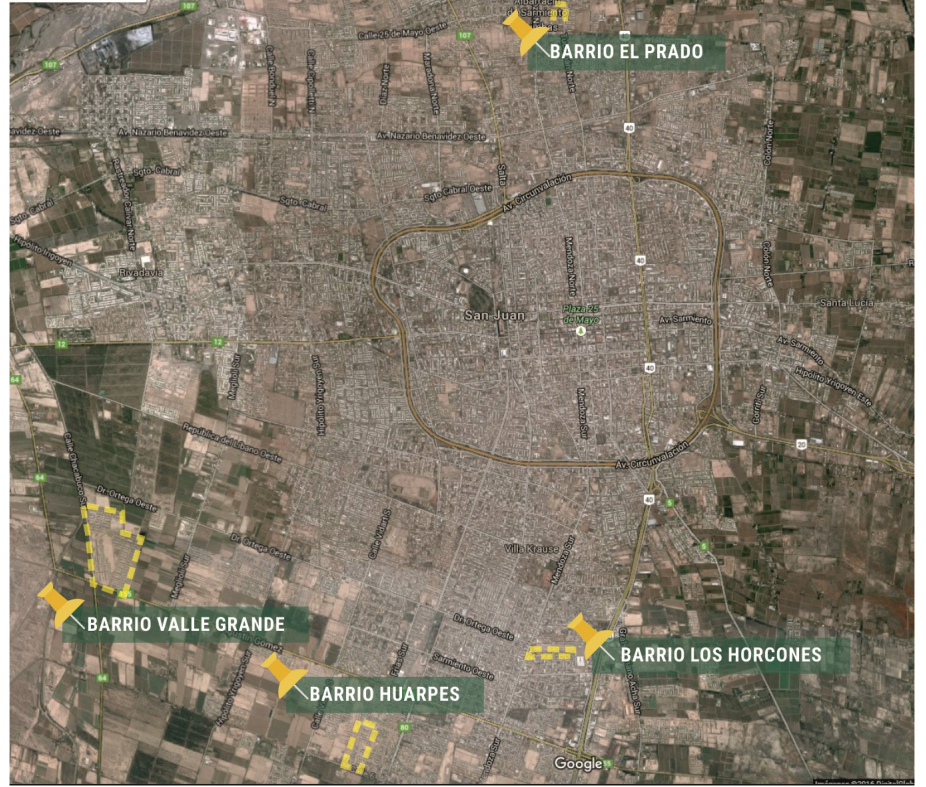

Figura 2. Distribución espacial de los Barrios analizados. Fuente: Elaboración de las autoras con base en Google Maps.

también las del emprendimiento urbano al que pertenecen. Como resultado, se obtiene que las emisiones de $\mathrm{CO}_{2}$ del hábitat social de una zona árido-sísmica son de 683.704,79 $\mathrm{kgCO}_{2} \mathrm{eq} / \mathrm{kWh}$. La materialización de las viviendas analizadas corresponde a la práctica constructiva generalizada, por tanto, si bien dista de ser una resolución sustentable, se la clasifica como "aceptable".

De los valores promedio, se extrae que el mayor porcentaje de emisiones se vincula con la EO. Si se correlaciona dicho valor con el de $\mathrm{El}_{\mathrm{T}}$, se obtiene que $\mathrm{El}_{\mathrm{T}}$ se corresponde con un $94,13 \%$ de $\mathrm{EO}$, en tanto para "fin de vida" la relación se establece en el orden del 0,04\%. Si se consideran las emisiones totales por vivienda, tendríamos que la EO posee una incidencia sobre el resultado final del $51.5 \%$, en tanto a la $\mathrm{El}_{\mathrm{T}}$ corresponde el $48,48 \%$ y al "fin de vida", el 0,02\%.

A los efectos de direccionar las estrategias de mejora y optimizar el ciclo de vida de los materiales constructivos de la vivienda social en zonas árido-sísmicas, se plantean cinco escenarios posibles: muy favorable, favorable, base, desfavorable y muy desfavorable. Al escenario "base" corresponden los valores promedio alcanzados en la MOM, mientras que los escenarios "muy favorable" y "favorable" presentan reducciones en las emisiones totales en el orden del $50^{15}$ y $25 \%$, respectivamente. Los escenarios "desfavorable" y "muy desfavorable" implican incrementos de $\mathrm{CO}_{2}$ del 25 y el 50\%. La Tabla 9 muestra los resultados obtenidos en cada caso.

[15] Esto, en coherencia con el objetivo propuesto para 2050 en el Acuerdo de París. Fuente: https://www. europapress.es/sociedad/medio-ambiente-00647/noticia-28-pactan-defender-reduccion-global-co2-50-cumbre-climaparis-20150918140207.html 


\begin{tabular}{|c|c|c|c|c|c|c|c|c|c|}
\hline Barrio & 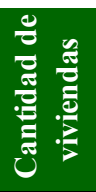 & CCI & $\mathbf{E I}_{\mathbf{T}}$ & Puntaje & EO & $\begin{array}{c}\text { Fin de } \\
\text { vida }\end{array}$ & $\begin{array}{l}\text { Emisiones } \\
\text { totales } \\
\text { vivienda } \\
\left.\text { (kg CO} \mathrm{C}_{2} \mathrm{eq}\right)\end{array}$ & $\begin{array}{c}\text { Emisiones } \\
\text { totales } \\
\text { barrio } \\
(\mathrm{kg} \mathrm{CO} \mathbf{e q})\end{array}$ & Tipo \\
\hline Los Horcones & 110 & 73693.66 & 340724.1 & 11.2 & 333227.8 & 125.99 & 674077.86 & 74148564.9 & 2 \\
\hline Valle Grande & 1000 & 73013.71 & 347373.5 & 11.2 & 405905.56 & 46.1011 & 753325.13 & 753325132.8 & 2 \\
\hline Huarpes & 286 & 65190.47 & 301126.5 & 11.2 & 345535.9 & 86.5906 & 646749 & 184970214.4 & 2 \\
\hline El Prado & 48 & 70996.17 & 336617.8 & 11.2 & 323778.6 & 270.808 & 660667.16 & 31712023.8 & 2 \\
\hline \multicolumn{2}{|c|}{ Valores promedio } & 70723.5 & 331460.5 & 11.2 & 352112 & 132.373 & 683704.79 & 261038984 & 2 \\
\hline
\end{tabular}

Tabla 8. Síntesis de los resultados por barrio. Fuente: Elaboración de las autoras.

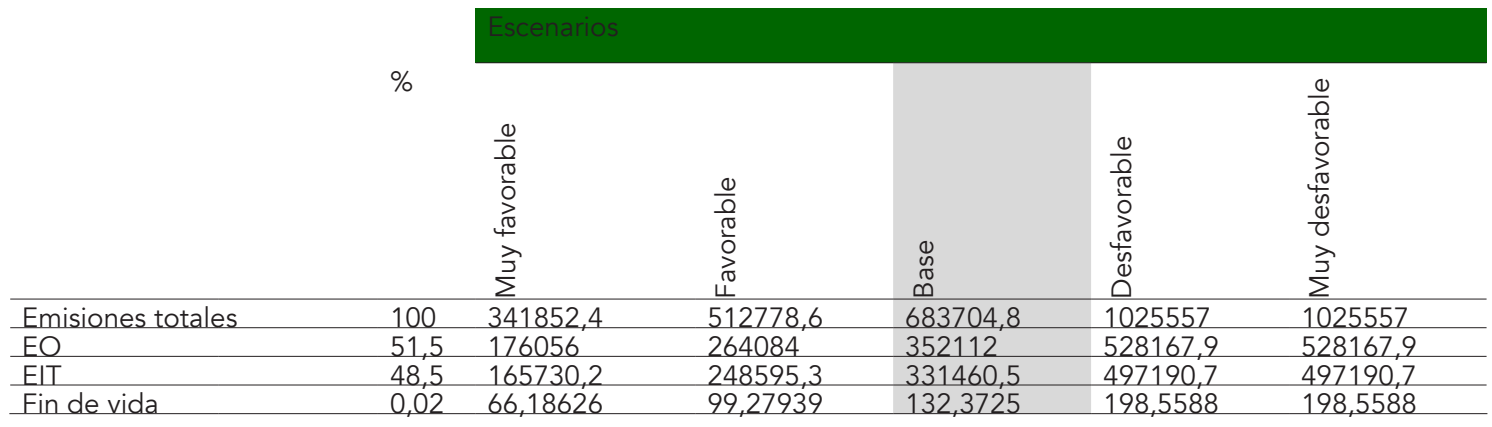

Tabla 9. Síntesis de los resultados por escenarios. Fuente: Elaboración de las autoras.

La MOM se ha diseñado con el fin de ser utilizada como una herramienta de apoyo a la decisión en instancias iniciales del proyecto arquitectónico, ya que su uso facilita la estimación rápida y expeditiva del perfil ambiental de la vivienda a lo largo de toda su vida útil. La manipulación predictiva de los datos de entrada de la MOM permite la selección de soluciones constructivas y de emplazamiento con menores impactos ambientales. Al respecto, la Tabla 9 establece la línea base de la práctica constructiva habitual del hábitat social en zona árido-sísmica, al tiempo que direcciona las modificaciones proyectuales, o bien, las estrategias de mejora, conforme a los valores límites establecidos para cada escenario.

\section{CONCLUSIONES}

La MOM se basa en la estimación temprana, a lo largo de su ciclo de vida, de las emisiones de $\mathrm{CO}_{2}$ asociadas a la materialización y emplazamiento de la vivienda social en zona árido-sísmica. A los efectos de facilitar la evaluación en etapa de diseño, se excluye la influencia en el análisis de carpinterías y revoques, dado que su inclusión excede los alcances de este estudio. No obstante, la MOM permite incorporar a futuro, el análisis de dichos parámetros, así como también otras opciones constructivas que resulten apropiadas y sean generalizables a zonas árido-sísmicas. Desde ese punto de vista, su uso aporta a la mejora continua de dichos sistemas constructivos.
En relación con los resultados alcanzados para los distintos casos de estudio analizados, se concluye que los criterios que sustentan la materialización de la vivienda social en la actualidad se encuentran fuertemente enraizados en variables de índole económica que desestiman parámetros ambientales y de confort higrotérmico. En este sentido, se observa que las soluciones constructivas son monótonas, reiterativas y generalizadas. Es decir, que distintos emplazamientos urbanos presentan las mismas resoluciones tecnológicas y funcionales, de manera que el perfil ambiental de las viviendas estudiadas no se corresponde con los avances normativos en sustentabilidad edilicia desarrollados tanto a nivel mundial como nacional. Así, optimizar los materiales constructivos en zonas áridosísmicas implica una mirada integral de la problemática habitacional en términos de ciclo de vida.

De igual forma, se detecta que dentro del hábitat social existente las estrategias de mejora posibles son de tipo comportamentales, o bien, de sistemas con mayor eficiencia energética. Al respecto, educar al usuario en materia de uso racional de la energía, eficiencia energética y ciclo de vida constituye un camino para empoderar a los mismos, en aras de que puedan ejercer su derecho a una vivienda digna y a un ambiente sano, equilibrado y apto para el desarrollo humano, tal como lo establecen los artículos 14bis y 41 de la Constitución Nacional Argentina. 


\section{FUTURAS INVESTIGACIONES}

Los resultados obtenidos se contrastarán, a futuro, con softwares específicos de LCA, tales como OPEN LCA y de análisis multiobjetivo. La aplicación de los mismos requiere de conocimientos cuya profundidad escapan a los alcances de esta investigación, además de requerir de bases de datos en relación con los impactos ambientales asociados a la edificación que, hasta el momento, no se han desarrollado para Argentina.

\section{AGRADECIMIENTOS}

Esta investigación se enmarca en el proyecto titulado "Optimización del Ciclo de Vida de los Materiales Constructivos de zonas árido-sísmicas" $1^{\circ}$ Etapa, Convocatoria 2016-2017, Res. N86/16-CD-FAUD y $2^{\circ}$ Etapa, Convocatoria 2017-2018, Res. N058/17-CD-FAUD. $\mathrm{Se}$ agradece al Instituto de Estudios en Arquitectura Ambiental (INEAA) por ser la institución en que se realizó la investigación y a la Facultad de Arquitectura, Urbanismo y Diseño (FAUD) de la Universidad Nacional de San Juan (UNSJ), por financiar la misma.

\section{REFERENCIAS BIBLIOGRÁFICAS}

ALI, Ahmed; NEGM, Adbelazim; BADY, Mahmoud e IBRAHIM, Mona. Environmental life cycle assessment of a residential building in Egypt: A case study, Procedia Technology [en línea], 2015, vol. 19, pp. 349-356. [Consultado 8 de agosto 2018]. Disponible en: https://core.ac.uk/download/pdf/82527681.pdf

ANTÓN VALLEJO, Asunción. Utilización del Análisis del ciclo de vida en la evaluación del impacto ambiental del cultivo bajo invernadero mediterráneo. Tesis Doctoral. Universitat Politècnica de Catalunya (UPC) (España), 2004.

BASBAGILL, John; FLAGER, Forest; LEPECH, Michel y FISCHER, Martin. Application of life-cycle assessment to early stage building design for reduced embodied environmental impacts, Building and Environment, 2013, vol. 60, pp. 81-92.

CARABAÑO, Rocío; BEDOYA, César y RUIZ, Diego. La metodología del análisis de ciclo de vida para la evaluación del impacto ambiental en el sector de la construcción: Estado del arte [en línea]. En: I Congreso Internacional sobre investigación en Construcción y Tecnología Arquitectónicas. Universidad Politécnica de Madrid. ETSAM. [Consultado 8 de octubre de 2018]. Disponible en: https://www.researchgate.net/ publication/263357247_La_metodologia_del_analisis_de_ciclo_ de_vida_para_la_evaluacion_del_impacto_ambiental_en_el_ sector_de_la_construccion_Estado_del_arte. 2014.

CARABAÑO, Rocío; HERNANDO, Susana; RUIZ, Diego y BEDOYA, César. Análisis de Ciclo de Vida (ACV) de los materiales de construcción para la evaluación de la sostenibilidad en la edificación: el caso de los materiales de aislamiento térmico, Revista de la Construcción [en línea], 2017, vol. 16, n 1, pp. 2232. DOI: http://dx.doi.org/10.7764/RDLC.16.1.22.
CARPINETTI Bruno y ESPONDA, Alejandra. Introducción al desarrollo sustentable. 2a ed. Buenos Aires: Universidad Nacional Arturo Jauretche, 2013.

CELEMÍN, Juan Pablo. El proceso analítico jerárquico en el marco de la evaluación multicriterio: un análisis comparativo, Geografía y Sistemas de Información Geográfica (GEOSIG), 2014, año 6, n 6, pp. 47-63.

CHACÓN, Mercedes Andrea y D'AMELIO, Aldana Florencia. Estudio comparativo del impacto ambiental de una vivienda convencional y de una vivienda sustentable en la etapa de la construcción. Avances y estado de situación en análisis de ciclo de vida y huellas ambientales en argentina. En: Actas del IV Encuentro Argentino de Ciclo de Vida y III Encuentro de la Red Argentina de Huella Hídrica - ENARCIV, 2015, pp. 73-75.

CORONA BELLOSTAS, Blanca. Análisis de Sostenibilidad del Ciclo de Vida de una Configuración innovadora de Tecnología Termosolar [en línea]. Tesis Doctoral. Universidad Politécnica de Madrid. Departamento de Ingeniería Química Industrial y Medio Ambiente, Escuela Técnica Superior de Ingenieros Industriales. [Consultado 8 de agosto 2018]. Disponible en: http://oa.upm.es/43813/1/ BLANCA_CARMEN_CORONA_BELLOSTAS.pdf. 2016.

DELGADO CASTILLO, Carlos y VELÁZQUEZ FLORES, Gerardo. Materiales de Construcción Sustentables en México: Políticas Públicas y Desempeño Ambiental. En: $X$ Seminario Itinerante del Campo Estratégico de Acción en Pobreza y Exclusión del Sistema Universitario Jesuita. México: Universidad Iberoamericana, 2012, pp. 1-53.

GARRIDO PIÑERO, Julia. Metodología de Evaluación y Minimización del Impacto Medioambiantal de tipologías residenciales de vivienda colectiva en la ciudad de Sevilla. Tesis Doctoral. Departamento de Construcciones arquitectónicas. Escuela Técnica Superior de Arquitectura. Universidad de Sevilla, 2015.

HURTADO TOSKANO, Bruno. El proceso de Análisis Jerárquico (AHP) como herramienta para la toma de decisiones en la Selección de Proveedores [en línea]. Tesis Digitales UNMSM. [Consultado 8 de agosto 2018]. Disponible en: http://sisbib.unmsm.edu.pe/bibvirtual/ monografias/basic/toskano_hg/contenido.htm. 2005.

IPCC. Fifth Assessment Report. Global Warming Potential Values [en línea], 2014. [Consultado 8 de agosto 2018]. Disponible en: http:// www.ghgprotocol.org/sites/default/files/ghgp/Global-WarmingPotential-Values\%20\%28Feb\%2016\%202016\%29_1.pdf.

IRAM 11601. Aislamiento térmico de edificios. Métodos de cálculo. Propiedades térmicas de los componentes y elementos de construcción en régimen estacionario. UNSJ. Biblioteca de la Facultad de Ingeniería. Instituto de Mecánica Aplicada. Norma. Argentina, 2002.

IRAM 11603. Acondicionamiento térmico de edificios. Clasificación bioambiental de la República Argentina. UNSJ. Biblioteca de la Facultad de Ingeniería. Instituto de Mecánica Aplicada. Norma. Argentina, 2012.

IRAM 11604. Aislamiento térmico de edificios. Verificación de sus condiciones higrotérmicas. Ahorro de energía en calefacción. Coeficiente volumétrico $G$ de pérdidas de calor. Cálculo y valores límites. UNSJ. Biblioteca de la Facultad de Ingeniería. Instituto de Mecánica Aplicada. Norma. Argentina, 2001. 
IRAM 11605. Acondicionamiento térmico de edificios. Condiciones de habitabilidad en edificios. Valores máximos de transmitancia térmica en cerramientos opacos. UNSJ. Biblioteca de la Facultad de Ingeniería. Instituto de Mecánica Aplicada. Norma. Argentina, 1996.

IRAM 11659-1. Aislamiento térmico de edificios. Verificación de sus condiciones higrotérmicas. Ahorro de energía en refrigeración. Parte 1: Vocabulario, definiciones, tablas y datos para determinar la carga térmica de refrigeración. UNSJ. Biblioteca de la Facultad de Ingeniería. Instituto de Mecánica Aplicada. Norma. Argentina, 2004.

IRAM 11659-2. Acondicionamiento térmico de edificios. Verificación de sus condiciones higrotérmicas. Ahorro de energía en refrigeración. Parte 2: Edificios para viviendas. UNSJ. Biblioteca de la Facultad de Ingeniería. Instituto de Mecánica Aplicada. Norma. Argentina, 2007.

IRAM 21931-1/12. Construcción sostenible. Marco de referencia para los métodos de evaluación del desempeño ambiental de las obras de construcción. Parte 1- Edificios. UNSJ. Biblioteca de la Facultad de Ingeniería. Instituto de Mecánica Aplicada. Norma. Argentina, 2012.

LÓPEZ-MESA, Belinda; PALOMERO CÁMARA, José; ORTEGA ZAPATA, Agustín y DEL AMO SANCHO, Alejandro. La rehabilitación y la mejora de la eficiencia energética de la vivienda social a examen, Monografías de la Revista Aragonesa de Administración Pública, 2013, vol. XV, pp. 283-319.

MARRERO, Madelyn; MARTíNEZ-ESCOBAR, Luna; MERCADER, Pilar y LEIVA, Carlos. Minimización del impacto ambiental en la ejecución de fachadas mediante el empleo de materiales reciclados, Informes de la Construcción [en línea], 2013, vol. 65, n 529, pp. 89-97. DOI: 10.3989/ic.11.034.

MUÑOZ SANGUINETTI, Claudia y QUIROZ ORTIZ, Francisco. Análisis de Ciclo de Vida en la determinación de la energía contenida y la huella de carbono en el proceso de fabricación del hormigón premezclado. Caso estudio planta productora Región del Bío-Bío, Chile, Revista Hábitat Sustentable, 2014, vol. $4, n^{\circ} 2$, pp. $16-25$.

QUISPE GAMBOA, Claudia N. Análisis de la Energía Incorporada y Emisiones de $\mathrm{CO}_{2}$ aplicado a viviendas unifamiliares de eficiencia energética [en línea]. Tesis de Maestría. Universidad Politécnica de Cataluña Escuela Técnica Superior de Arquitectura de Barcelona Máster en Arquitectura, Energía y Medio Ambiente, 2016. [Consultado 8 de agosto 2018]. Disponible en: https:// wwwaie.webs.upc.edu/maema/wp-content/uploads/2016/10/ Quispe-Gamboa-Claudia-Nataly.pdf

QUISPE LOYOLA, César. Aplicación del proceso analítico jerárquico $(A H P)$ en la selección de un marco de referencia para gestionar los proyectos de una empresa consultora. Tesina. Universidad Nacional Mayor de San Marcos Facultad de Ciencias Matemáticas E.A.P. de Investigación Operativa. Lima, Perú, 2017.

ROS GARCÍA, Juan y SANGLIER CONTRERAS, Gastón. Análisis del Ciclo de Vida de una Unidad Prototipo de Vivienda de Emergencia. La búsqueda del impacto nulo. Informes de la Construcción [en línea], 2017, vol. 69, n 547, p. e211. DOI: http://dx.doi.org/10.3989/ic.16.035. 2017.
RÖCK, Martin; HOLLBERG, Alexander; HABERT, Guillaume y PASSER, Alexander. LCA and BIM: Integrated assessment and visualization of building elements' embodied impacts for design guidance in early stages. En: 25th CIRP Life Cycle Engineering (LCE) Conference, 2018, Copenhagen, Denmark Procedia CIRP 69 [en línea],2018, pp.218-223. [Consultado8deagosto2018]. Disponible en: $\quad$ https://ac.els-cdn.com/S2212827117308636/1-s2.0S2212827117308636-main.pdf?_tid=61036bbc-08a3-4bc0-902a 